\title{
Not on the right trach
}

Tracheostomies are a common procedure, typically carried out in the intensive care setting. Performed for upper airway obstruction, airway protection or prolonged endotracheal intubation, tracheostomies are a life-saving intervention yet are associated with significant morbidity and mortality. In 2011, the National Audit Project reported $70 \%$ of airway incidents and $60 \%$ of airway related deaths within the intensive care unit (ICU) related to tracheostomy complications. ${ }^{1}$ Often these difficulties stemmed from airway aftercare rather than problems during insertion. Given the potential for adverse patient outcomes, the Intensive Care Society sought to readdress the issues surrounding tracheostomy care by generating staff guidance which stated that 'All staff working in clinical locations where tracheostomy patients are managed must be competent to assess and initiate management in the event of an airway emergency. ${ }^{2}$

While the rationale for improving knowledge regarding tracheostomy management is prudent, the reality is that staff education is often limited to the critical care environment with the importance of such training becoming lost on the medical wards. Yet, worsening bed pressures have resulted in an increasing number of patients with temporary tracheostomies being discharged from the ICU to medical wards prior to decannulation. Moreover, when patients with tracheostomies become critically unwell, junior doctors are typically the first point of contact meaning they are increasingly being confronted with complex tracheostomy patients, often without the necessary training. It is therefore unsurprising that the 2014 UK National Confidential Enquiry into Patient Outcomes and Death identified significant safety issues in the hospital care of patients with tracheostomies. ${ }^{3}$

Previously, Findlay and Abbas attempted to assess junior doctor confidence in tracheostomy emergencies noting that the majority felt apprehensive and ill-equipped to deal with such scenarios. ${ }^{4}$ Referencing this work, we noted a similar trend in junior doctors working on respiratory and acute medical wards in a Belfast hospital. Specifically, 12 of the $18(66.7 \%)$ respondents reported feeling both unable to manage an acutely unwell patient with a tracheostomy or a tracheostomy airway emergency. This was despite $55.6 \%$ having received previous undergraduate training. Evidently, there exists a need for improved junior doctor tracheostomy training yet, critically, all those surveyed were expected to work as members of the cardiac arrest team when on call, making the lack of confidence in this area alarming and potentially dangerous.

When one considers the potential for harm, it is somewhat perplexing that tracheostomy airway emergencies do not form part of the Resuscitation Council's formal Advanced Life Support (ALS) training. Acknowledging the Resuscitation Council's role in creating bedside algorithms which document the correct management of tracheostomy airway emergencies as part of the National Tracheostomy Safety Project, we argue that these form only a small part of what must be a wider educational process. ${ }^{5}$ In an emergency, when high stress levels may cloud thinking or the aforementioned algorithm is not immediately available, the ability to draw upon previous experience in tracheostomy emergencies would be invaluable in improving junior doctors knowledge regarding how they should proceed, thus enhancing patient safety outcomes.

Moving forward, we believe that in order to generate meaningful improvements in patient safety for tracheostomy patients a multidimensional approach to training is required. The combined effects of undergraduate training, mandatory postgraduate training (within trust induction programmes) and the incorporation of tracheostomy emergencies into future ALS courses, are likely to improve junior doctors' capabilities and confidence in managing these complex patients.

EMMA KEELAN

ST5 in respiratory medicine, Royal Victoria Hospital, Belfast

NIALLA DOHERTY

FY1, Royal Victoria Hospital, Belfast

\section{References}

1 Cook T, Woodall N, Frerk C (eds). 4th National Audit Project of The Royal College of Anaesthetists and The Difficult Airway Society: Major complications of airway management in the United Kingdom. London: Royal College of Anaesthetists, 2011. www.rcoa. ac.uk/system/files/CSQ-NAP4-Full.pdf [Accessed 10 October 2019].

2 Intensive Care Society. Standards for the care of adult patients with a temporary tracheostomy: Standards and guidelines. ICS, 2014. www.theawsomecourse.co.uk/ICS/ICS\% 20Tracheostomy \% 20standards \% 20(2014).pdf [Accessed 09 October 2019].

3 National Confidential Enquiry into Patient Outcomes and Death. On the right trach? A review of the care received by patients who underwent a tracheostomy. NCEPOD, 2014. www.ncepod.org. uk/2014report1/downloads/OnTheRightTrach_FullReport.pdf [Accessed 12 October 2019].

4 Findlay G, Abbas Y. Management of tracheostomy-related emergencies: An audit of junior doctors' knowledge and skills. J Cardiothorac Surg 2015;10(Suppl 1):A278.

5 Resuscitation Council. The National Tracheostomy Safety Project. Resuscitation Council newsletter 2012;23:8-9. www.resus.org.uk/ archive/archived-newsletters [Accessed 14 October 2019].

Address for correspondence: Dr Emma Keelan, Royal Victoria Hospital, 274 Grosvenor Rd, Belfast BT12 6BA, UK. Email: ekeelan01@qub.ac.uk 